\title{
Power of TOF-SIMS Tandem MS Imaging: Industrial Problem Solving to Investigating Nature
}

\author{
Andrew Giordani, David Carr, Ashley Ellsworth, Scott Bryan and Gregory Fisher
}

Physical Electronics, Chanhassen, Minnesota, United States

Time-of-Flight Secondary Ion Mass Spectrometry (TOF-SIMS) is powerful analytical technique that has been heavily utilized to analyze surface chemistries due to its very shallow sampling depth (10-20 ̊), high chemical sensitivity, and ability to provide elemental and molecular information. Additionally, TOFSIMS can produce chemical images with high spatial resolution $(<70 \mathrm{~nm})$. Over the past 40 years TOFSIMS has evolved immensely from primarily being used in universities for fundamental studies to an invaluable tool to solve industrial problems. However, historically, TOF-SIMS has been hampered by the ability to confidently identify high mass secondary ions $(>140 \mathrm{~m} / \mathrm{z})$ with high mass accuracy (< $10 \mathrm{ppm}$ ) [1]. This problem has only become worse with use of cluster ion beams (e.g. Au3+, C60+, Bi3+, and Ar gas cluster) which significantly improves secondary ion yields for higher mass fragments. To overcome this problem, Physical Electronics has developed a revolutionary TOF-SIMS tandem MS method specifically designed to unambiguously identify the composition of high mass secondary ions [2]. This method allows for MS/MS spectra (MS2) of select secondary ions of interest (precursor ions) to be acquired in parallel with conventional TOF-SIMS spectra (MS1); both being simultaneously generated from the same analytical volume. As the MS2 spectra are predominantly generated from single molecular ions, they are much cleaner and simpler than conventional MS1 spectra which usually contain secondary ions from multiple chemical species. The MS/MS fragmentation spectrum (MS2) is used to identify the composition of the precursor ion by either identification of the fragmented ions and/or comparison to spectra contained in on-line databases. This talk will focus on the power of TOF-SIMS tandem MS imaging to solve industrial problems, such as quality control, failure analysis, and forensics, as well as, a correlative TEM and TOF-SIMS study to characterize the wing of a Damselfly, a complex biological sample.

\section{References}

[1] F. M. Green, I. S. Gilmore, and M. P. Seah, J. Am. Soc. Mass Spectrom., 17, 514 (2006).

[2] G.L. Fisher, J.S. Hammond, P.E. Larson, S.R. Bryan, and R.M.A. Heeren, J. Vac. Sci. Technol. B, 34 (2016). 